ANNALES

POLONICI MATHEMATICI

$95.2(2009)$

\title{
Growth of solutions of a class of complex differential equations
}

\author{
by Ting-Bin CAO (Nanchang)
}

\begin{abstract}
The main purpose of this paper is to partly answer a question of L. Z. Yang [Israel J. Math. 147 (2005), 359-370] by proving that every entire solution $f$ of the differential equation $f^{\prime}-e^{P(z)} f=1$ has infinite order and its hyperorder is a positive integer or infinity, where $P$ is a nonconstant entire function of order less than $1 / 2$. As an application, we obtain a uniqueness theorem for entire functions related to a conjecture of Brück [Results Math. 30 (1996), 21-24].
\end{abstract}

1. Introduction and main results. In this paper, we will use the standard notations of Nevanlinna's value distribution theory (see [12], [19]). During the last ten years many papers have been devoted to the study of the growth of solutions of complex differential equations (see [15]). By making use of the properties of the logarithmic derivative, it is easy to see that if $A(z)$ is a transcendental entire function, then every nonzero solution $f$ of the equation $f^{(k)}+A(z) f=0$ is an entire function of order $\sigma(f)=\infty$. For the corresponding nonhomogeneous linear differential equation

$$
f^{(k)}+A(z) f=F(z) .
$$

Chen and Gao (see [5]) proved that if $A$ is a transcendental entire function and if $F \not \equiv 0$ is an entire function of finite order, then every solution $f$ satisfies $\sigma(f)=\lambda(f)=\infty$, with at most one possible exception. Here $\sigma(f)$ and $\lambda(f)$ denote the order of $f$ and the convergence exponent of zeros of $f$, respectively.

Thus an interesting problem arises: What conditions on $A$ and $F$ guarantee that every solution $f$ of (1) has infinite order?

G. G. Gundersen and L. Z. Yang obtained the following result related to a conjecture of R. Brück [4].

2000 Mathematics Subject Classification: Primary 34M10; Secondary 30D35.

Key words and phrases: hyperorder, differential equation, entire function. 
TheOREM 1.1 ([11]). Let $P$ be a nonconstant polynomial. Then every solution $f$ of the differential equation $f^{\prime}-e^{P(z)} f=1$ is an entire function of infinite order.

The hyperorder ([23]) of a meromorphic function $f$ is defined by

$$
\sigma_{2}(f)=\limsup _{r \rightarrow \infty} \frac{\log ^{+} \log ^{+} T(r, f)}{\log r} .
$$

In [21] (see also [22]), L. Z. Yang raised the question below, and proved that if, in Theorem 1.1; $P$ is a nonconstant entire function then the hyperorder of $f$ is a positive integer or infinity with at most one exception. However, we do not know whether the exceptional solution exists or not.

Question ([21] or [22]). Is it true that if $P$ is a nonconstant entire function then the hyperorder of $f$ satisfying the equation of Theorem 1.1 is a positive integer or infinity?

The main purpose of this paper is to deal with this question. We will prove that if the order of $P$ is less than $1 / 2$, then there does not exist an exceptional solution. The idea is taken from [6], [7] or [9], and it is very different from Yang [22]. Now we show our main result which improves Theorem 1.1 and some results in [18], [20], [9].

TheOrem 1.2. Let $P$ be a nonconstant entire function, let $Q$ be a nonzero polynomial, and let $f$ be any entire solution of the differential equation

$$
f^{(k)}-e^{P(z)} f=Q(z) \quad(k \in \mathbb{N}) .
$$

If $P$ is a polynomial, then $f$ has infinite order and its hyperorder $\sigma_{2}(f)$ is a positive integer not exceeding the degree of $P$. If $P$ is transcendental with order less than $1 / 2$, then the hyperorder of $f$ is infinite.

Theorem 1.2 immediately yields the following corollary, which answers the above question when $P$ is a nonconstant entire function with order less than $1 / 2$.

COROLlary 1.1. Let $P$ be a nonconstant entire function with $\sigma(P)<$ $1 / 2$. Then every entire solution $f$ of the differential equation $f^{\prime}-e^{P(z)} f=1$ has infinite order and its hyperorder is a positive integer or infinity.

Next, we shall show an interesting result when $P$ is a gap series, which also partly answers the question of L. Z. Yang.

THEOREM 1.3. Let $P(z)=\sum_{\nu=0}^{\infty} c_{\nu} z^{n_{\nu}}$ be a nonconstant entire function of finite lower order with Fabry gaps, that is,

$$
\nu / n_{\nu} \rightarrow 0 \quad \text { as } \nu \rightarrow \infty .
$$


Then every entire solution $f$ of the differential equation $f^{\prime}-e^{P(z)} f=1$ has infinite order and its hyperorder is a positive integer or infinity.

2. Lemmas. For the proof of our result, we need the following lemmas.

Lemma 2.1 ([8]). Let $g$ be an entire function of infinite order with hyperorder $\sigma_{2}(g)$ and let $\nu(r, g)$ be the central index of $g$. Then

$$
\limsup _{r \rightarrow \infty} \frac{\log ^{+} \log ^{+} \nu(r, g)}{\log r}=\sigma_{2}(g) .
$$

LEMMA $2.2([7])$. Let $f$ be an entire function of infinite order with hyperorder $\sigma_{2}(f)=\alpha<\infty$, and let $E \subset[1, \infty)$ be a set of finite logarithmic measure. If $\alpha>0$, then there exists a sequence $\left\{z_{k}=r_{k} e^{i \theta_{k}}\right\}$ such that $\left|f\left(z_{k}\right)\right|=M\left(r_{k}, f\right), \theta_{k} \in[0,2 \pi), \lim _{k \rightarrow \infty} \theta_{k}=\theta_{0} \in[0,2 \pi)$, and for any given $\varepsilon>0$,

$$
\exp \left\{r_{k}^{\alpha-\varepsilon}\right\}<\nu\left(r_{k}, f\right)<\exp \left\{r_{k}^{\alpha+\varepsilon}\right\}
$$

for all sufficiently large $r_{k} \notin E$. If $\alpha=0$, then there also exists such a sequence, and for any large $M>0$,

$$
\nu\left(r_{k}, f\right)>r_{k}^{M}
$$

for all sufficiently large $r_{k} \notin E$.

Lemma 2.3 ([16]). Let $P(z)=b_{n} z^{n}+b_{n-1} z^{n-1}+\cdots+b_{0}$, where $n$ is a positive integer and $b_{n}=\alpha_{n} e^{i \theta_{n}}, \alpha_{n}>0, \theta_{n} \in[0,2 \pi)$. For any given $\varepsilon$ with $0<\varepsilon<\pi /(4 n)$, we introduce $2 n$ open angles

$$
S_{j}:-\frac{\theta_{n}}{n}+(2 j-1) \frac{\pi}{2 n}+\varepsilon<\theta<-\frac{\theta_{n}}{n}+(2 j+1) \frac{\pi}{2 n}-\varepsilon,
$$

where $j=0,1, \ldots, 2 n-1$. Then there exists a positive number $R=R(\varepsilon)$ such that for $|z|=r>R$,

$$
\operatorname{Re}\{P(z)\}>\alpha_{n}(1-\varepsilon) \sin (n \varepsilon) r^{n}
$$

if $z \in S_{j}$ with $j$ even, while

$$
\operatorname{Re}\{P(z)\}<-\alpha_{n}(1-\varepsilon) \sin (n \varepsilon) r^{n}
$$

if $z \in S_{j}$ with $j$ odd.

Now for any given $\theta \in[0,2 \pi)$, if $\theta \neq-\theta_{n} / n+(2 j-1) \pi /(2 n)(j=$ $0,1, \ldots, 2 n-1)$, then for $\varepsilon$ sufficiently small, there exists $j \in\{0,1, \ldots, 2 n-1\}$ such that $\theta \in S_{j}$.

For any $E \subset \mathbb{R}$, define

$$
\begin{aligned}
& \underline{\log \operatorname{dens}}(E)=\liminf _{r \rightarrow \infty} \frac{\int_{1}^{r}\left(\chi_{E}(t) / t\right) d t}{\log r}, \\
& \overline{\log \operatorname{dens}}(E)=\limsup _{r \rightarrow \infty} \frac{\int_{1}^{r}\left(\chi_{E}(t) / t\right) d t}{\log r} .
\end{aligned}
$$


Lemma 2.4 ([2]). Let $f$ be an entire function of order $\sigma(f)=\sigma<1 / 2$, and define

$$
A(r)=\inf _{|z|=r} \log |f(z)|, \quad B(r)=\sup _{|z|=r} \log |f(z)| .
$$

If $\sigma<\alpha<1$, then

$$
\underline{\log \operatorname{dens}}\{r: A(r)>(\cos \pi \alpha) B(r)\} \geq 1-\sigma / \alpha .
$$

Lemma $2.5([3])$. Let $f$ be an entire function with lower order $\mu(f)=$ $\mu<1 / 2$ and $\mu(f)<\sigma(f)=\sigma$. If $\mu \leq \delta<\min (\sigma, 1 / 2)$ and $\delta<\alpha<1 / 2$, then

$$
\overline{\log \operatorname{dens}}\left\{r: A(r)>(\cos \pi \alpha) B(r)>r^{\alpha}\right\}>c(\sigma, \delta, \alpha),
$$

where $c(\sigma, \delta, \alpha)$ is a positive constant depending only on $\sigma, \delta$ and $\alpha$.

Lemma $2.6([10])$. Let $f$ be a transcendental meromorphic function of finite order $\sigma$. Let $\varepsilon>0$ be a constant, and $k$ and $j$ be integers satisfying $k>j \geq 0$. Then:

(a) There exists a set $E_{1} \subset(1, \infty)$ of finite logarithmic measure such that for all $z$ satisfying $|z| \notin E_{1} \cup[0,1]$, we have

$$
\left|\frac{f^{(k)}(z)}{f^{(j)}(z)}\right| \leq|z|^{(k-j)(\sigma-1+\varepsilon)} .
$$

(b) There exists a set $E_{2} \subset[0,2 \pi)$ of linear measure zero such that if $\theta \in[0,2 \pi) \backslash E_{2}$, then there is a constant $R=R(\theta)>0$ such that (3) holds for all $z$ satisfying $\arg z=\theta$ and $R \leq|z|$.

Lemma $2.7([1])$. Let $g:[0, \infty) \rightarrow R$ and $h:[0, \infty) \rightarrow R$ be nondecreasing functions such that $g(r) \leq h(r)$ off an exceptional set $E_{2}$ of finite linear measure. Then for any $\alpha>1$, there exists $r_{0}$ such that $g(r) \leq h(\alpha r)$ for all $r>r_{0}$.

Lemma $2.8([10])$. Let $f$ be a transcendental meromorphic function. Let $\alpha>1$ be a constant, and $k$ and $j$ be integers satisfying $k>j \geq 0$. Then:

(a) There exists a set $E_{1} \subset(1, \infty)$ of finite logarithmic measure, and a constant $C>0$ such that for all $z$ satisfying $|z| \notin E_{1} \cup[0,1]$, we have (with $r=|z|$ )

$$
\left|\frac{f^{(k)}(z)}{f^{(j)}(z)}\right| \leq C\left[\frac{T(\alpha r, f)}{r}(\log r)^{\alpha} \log T(\alpha r, f)\right]^{k-j} .
$$

(b) There exists a set $E_{2} \subset[0,2 \pi)$ of linear measure zero such that if $\theta \in[0,2 \pi) \backslash E_{2}$, then there is a constant $R=R(\theta)>0$ such that (4) holds for all $z$ satisfying $\arg z=\theta$ and $R \leq|z|$. 
Lemma 2.9. Let $P$ be a nonconstant polynomial and let $Q$ be a nonzero polynomial. Then every solution $f$ of the differential equation (2) is an entire function of infinite order.

Proof. It is well known that $f$ must be an entire function. It is easy to see that $f$ is transcendental. We shall prove that $f$ is of infinite order. Indeed, otherwise by Lemma 2.6(b), there exists a set $E \subset[0,2 \pi)$ of linear measure zero such that for any $\operatorname{ray} \arg z=\theta \in[0,2 \pi) \backslash E$ and any given $0<\varepsilon<1$, there is an $R>0$ such that

$$
\left|\frac{f^{(k)}\left(r e^{i \theta}\right)}{f\left(r e^{i \theta}\right)}\right| \leq|z|^{k(\sigma(f)-1+\varepsilon)}
$$

for $|z|=r>R$. Set

$$
P(z)=b_{n} z^{n}+b_{n-1} z^{n-1}+\cdots+b_{0}, \quad b_{n} \neq 0,
$$

where $n \in \mathbb{N}$ and $b_{n}=\alpha_{n} e^{i \theta_{n}}, \alpha_{n}>0, \theta_{n} \in[0,2 \pi)$. By Lemma 2.3, if $\theta \neq-\theta_{n} / n+(2 j-1) \pi /(2 n)(j=0,1, \ldots, 2 n-1)$, then for sufficiently large $r$, we have

$$
\operatorname{Re}\{P(z)\}>\alpha_{n \theta} r^{n} \quad \text { or } \operatorname{Re}\{P(z)\}<-\alpha_{n \theta} r^{n},
$$

where $\alpha_{n \theta}$ is a positive constant. Now take

$$
\arg z=\theta \in[0,2 \pi) \backslash\left(E \cup \bigcup_{j=0}^{2 n-1}\left\{-\frac{\theta_{n}}{n}+(2 j-1) \frac{\pi}{2 n}\right\}\right) .
$$

CASE (i): If $\operatorname{Re}\{P(z)\}>\alpha_{n \theta} r^{n}$, then by (5),

$$
\left|\frac{f^{(k)}\left(r e^{i \theta}\right)}{f\left(r e^{i \theta}\right)}\right| \frac{1}{r^{k(\sigma(f)+M)}} \rightarrow 0, \quad \frac{\left|Q\left(r e^{i \theta}\right)\right|}{r^{k(\sigma(f)+M)}} \rightarrow 0, \quad \frac{\left|e^{P\left(r e^{i \theta}\right)}\right|}{r^{k(\sigma(f)+M)}} \rightarrow \infty,
$$

as $r \rightarrow \infty$, where $M$ is a constant with $M>\max \{\operatorname{deg}(Q) / k-\sigma(f), 2\}$. From (2),

$$
\frac{f^{(k)}}{f}-e^{P(z)}=\frac{Q(z)}{f} .
$$

It follows from (8) and (7) that

$$
\left|f\left(r e^{i \theta}\right)\right| \rightarrow 0 .
$$

CASe (ii): If $\operatorname{Re}\{P(z)\}<-\alpha_{n \theta} r^{n}$, then by (2) we have

$$
1-e^{P\left(r e^{i \theta}\right)} \frac{f\left(r e^{i \theta}\right)}{f^{(k)}\left(r e^{i \theta}\right)}=\frac{Q\left(r e^{i \theta}\right)}{f^{(k)}\left(r e^{i \theta}\right)} .
$$

Now we assert that $\left|f^{(k)}\left(r e^{i \theta}\right)\right| \leq 2\left|q_{s}\right| r^{\operatorname{deg}(Q)}$ on $\arg z=\theta$, where we set $Q(z)=q_{s} z^{s}+q_{s-1} z^{s-1}+\cdots+q_{0}$. If it is not true, then there exists a sequence $\left\{r_{m}\right\}$ such that

$$
2\left|q_{s}\right| r_{m}^{\operatorname{deg}(Q)}<\left|f^{(k)}\left(r e^{i \theta}\right)\right|=M\left(r_{m}, f^{(k)}\left(r e^{i \theta}\right), \theta\right),
$$


where

$$
M\left(r_{m}, f^{(k)}\left(r e^{i \theta}\right), \theta\right):=\max \left\{\left|f^{(k)}\left(r e^{i \theta}\right)\right|: 0 \leq r \leq r_{m}, \arg z=\theta\right\} .
$$

From

$$
f^{(k-1)}\left(r_{m} e^{i \theta}\right)=f^{(k-1)}(0)+\int_{0}^{r_{m} e^{i \theta}} f^{(k)}(u) d u
$$

we have

$$
\left|f^{(k-1)}\left(r_{m} e^{i \theta}\right)\right| \leq\left|f^{k-1}(0)\right|+r_{m}\left|f^{(k)}\left(r_{m} e^{i \theta}\right)\right|
$$

Again by

$$
\begin{aligned}
f^{(k-2)}\left(r_{m} e^{i \theta}\right) & =f^{(k-2)}(0)+\int_{0}^{r_{m} e^{i \theta}} f^{(k-1)}(u) d u \\
& =f^{(k-2)}(0)+\int_{0}^{r_{m} e^{i \theta}}\left(f^{(k-1)}(0)+\int_{0}^{u} f^{(k)}(s) d s\right) d u
\end{aligned}
$$

we have

$$
\left|f^{(k-2)}\left(r_{m} e^{i \theta}\right)\right| \leq\left|f^{(k-2)}(0)\right|+r_{m}\left|f^{(k-1)}(0)\right|+r_{m}^{2}\left|f^{(k)}\left(r_{m} e^{i \theta}\right)\right| .
$$

By induction we obtain

$$
\begin{aligned}
\left|f\left(r_{m} e^{i \theta}\right)\right| & \leq \sum_{j=0}^{k-1} r_{m}^{j}\left|f^{(j)}(0)\right|+r_{m}^{k}\left|f^{(k)}\left(r_{m} e^{i \theta}\right)\right| \\
& =(1+o(1)) r_{m}^{k}\left|f^{(k)}\left(r_{m} e^{i \theta}\right)\right|
\end{aligned}
$$

and hence

$$
\left|\frac{f\left(r_{m} e^{i \theta}\right)}{f^{(k)}\left(r_{m} e^{i \theta}\right)}\right| \leq(1+o(1)) r_{m}^{k}
$$

By the assumption $\operatorname{Re}\{P(z)\}<-\alpha_{n \theta} r^{n}$, together with (11) and (12), we have

$$
\left|e^{P\left(r_{m} e^{i \theta}\right)} \frac{f\left(r_{m} e^{i \theta}\right)}{f^{(k)}\left(r_{m} e^{i \theta}\right)}\right| \leq(1+o(1)) r_{m}^{k} e^{-\alpha_{n \theta} r_{m}^{n}}
$$

and

$$
\left|\frac{Q\left(r_{m} e^{i \theta}\right)}{f^{(k)}\left(r_{m} e^{i \theta}\right)}\right| \leq \frac{\left(\left|q_{s}\right|+o(1)\right) r_{m}^{\operatorname{deg}(Q)}}{2\left|q_{s}\right| r_{m}^{\operatorname{deg}(Q)}} \rightarrow \frac{1}{2} \quad(m \rightarrow \infty) .
$$

Now (13) and (14) contradict (10). This implies that

$$
\left|f\left(r e^{i \theta}\right)\right| \leq(1+o(1)) 2\left|q_{s}\right| r^{k+\operatorname{deg}(Q)}
$$

on $\arg z=\theta$.

Since the linear measure of $E \cup \bigcup_{j=0}^{2 n-1}\left\{-\theta_{n} / n+(2 j-1) \pi /(2 n)\right\}$ is equal to 0 , and since we assume that $f$ is of finite order, it can be deduced 
from (9), (15), the Phragmén-Lindelöf theorem (see [16, pp. 270-271]) and Liouville's theorem that $f$ must be a polynomial. This contradicts $(2)$ which implies that $f$ is transcendental. This proves that the order of $f$ is infinite.

Lemma $2.10([17])$. Let $f$ be an entire function of finite lower order $\mu(f)$. Suppose that $0<\varepsilon<1$ and

$$
f(z)=\sum c_{k} z^{n_{k}}
$$

with $\left\{n_{k}\right\}$ an increasing sequence of nonnegative integers for which

$$
k / n_{k} \rightarrow 0 \quad(k \rightarrow \infty) .
$$

Then for a set of radii $r$ of infinite logarithmic measure,

$$
\log L(r)>(1-\varepsilon) \log M(r),
$$

where $L(r)=L(r, f)$ is the minimum modulus of $f$ on $|z|=r$ and $M(r)=$ $M(r, f)$ is the maximum modulus of $f$ on $|z|=r$.

3. Proof of Theorem 1.2. We split the proof into two cases as follows. First, suppose that $P$ is a nonconstant polynomial

$$
P(z)=b_{n} z^{n}+b_{n-1} z^{n-1}+\cdots+b_{0}, \quad b_{n} \neq 0 .
$$

Then by Lemma 2.9, any solution $f$ of (2) is of infinite order. By the WimanValiron theory (see [13] or [15]), there exists a set $E \subset[1, \infty)$ of finite logarithmic measure such that for $|z|=r \notin[0,1] \cup E$, and $|f(z)|=M(r, f)$, we have

$$
\frac{f^{(k)}(z)}{f(z)}=\left(\frac{\nu(r, f)}{z}\right)^{k}(1+o(1)) .
$$

Substituting (16) into (2), we get

$$
\left(\frac{\nu(r, f)}{z}\right)^{k}(1+o(1))=e^{P(z)}+\frac{Q(z)}{f(z)}
$$

Set $b_{n}=\alpha_{n} e^{i \vartheta_{n}}, \alpha_{n}>0$, and $\vartheta_{n} \in[0,2 \pi)$. Since the order of $f$ is infinite and since $Q(z)-Q^{\prime}(z)$ is a polynomial, for sufficiently large $|z|=r$ and for any given $\varepsilon>0$, Lemma 2.1, Lemma 2.7 and (17) imply that $\sigma_{2}(f) \leq t \leq$ $\operatorname{deg}(P)=n$ when we choose $z$ with $|z|=r \notin[0,1] \cup E$, and $|f(z)|=M(r, f)$, where the integer $t$ satisfies $0 \leq t \leq n$ and $\operatorname{Re}\left\{b_{n} z^{n}\right\}=\cdots=\operatorname{Re}\left\{b_{t+1} z^{t+1}\right\}$ $=0$ and $\operatorname{Re}\left\{b_{t} z^{t}\right\} \neq 0$.

Let $E \subset[1, \infty)$ have finite logarithmic measure. By Lemma 2.2, if $\delta>0$ then there exists a sequence $\left\{z_{m}=r_{m} e^{i \theta_{m}}\right\}$ such that $\left|f\left(z_{m}\right)\right|=M\left(r_{m}, f\right)$, $\theta_{m} \in[0,2 \pi), \lim _{m \rightarrow \infty} \theta_{m}=\theta_{0} \in[0,2 \pi)$, and for any given $0<3 \varepsilon<$ $\min \{\delta, n-\delta, \pi /(4 n)\}$,

$$
\exp \left\{r_{m}^{\delta-\varepsilon}\right\}<\nu\left(r_{m}, f\right)<\exp \left\{r_{m}^{\delta+\varepsilon}\right\}
$$


for all sufficiently large $r_{m} \notin E$; if $\delta=0$ then there also exists such a sequence, and for $0<3 \varepsilon<\pi /(4 n)$ and any large $M_{1}>0$,

$$
\nu\left(r_{m}, f\right)>r_{m}^{M_{1}}
$$

for all sufficiently large $r_{m} \notin E$. It follows from (17) that for $z_{m}=r_{m} e^{i \theta_{m}}$ and $\left|f\left(z_{m}\right)\right|=M\left(r_{m}, f\right)$, we have

$$
\left(\frac{\nu\left(r_{m}, f\right)}{z_{m}}\right)^{k}(1+o(1))=e^{P\left(z_{m}\right)}+o(1) .
$$

By Lemma 2.3, there are $2 n$ open angles for $\varepsilon$ as above,

$$
\begin{aligned}
S_{j}:-\frac{\vartheta_{n}}{n}+(2 j-1) \frac{\pi}{2 n}+\varepsilon<\theta<-\frac{\vartheta_{n}}{n}+ & (2 j+1) \frac{\pi}{2 n}-\varepsilon, \\
& (j=0,1, \ldots, 2 n-1) .
\end{aligned}
$$

For the above $\theta_{0}$, we now consider the following three cases.

CASE (i): $\theta_{0} \in S_{j}$ with $j$ odd. Since $S_{j}$ is an open set and $\lim _{m \rightarrow \infty} \theta_{m}$ $=\theta_{0}$, there is $M_{2}>0$ such that $\theta_{m} \in S_{j}$ when $m>M_{2}$, and by Lemma 2.3,

$$
\operatorname{Re}\left\{P\left(r_{m} e^{i \theta_{m}}\right)\right\}<-d r_{m}^{n},
$$

where $d=\alpha_{n}(1-n \varepsilon) \sin (n \varepsilon)>0$. If $\delta>0$, then from $3 \varepsilon<\delta$ and (18), (20), (22), we obtain

$$
\exp \left\{k r_{m}^{\delta-\varepsilon}\right\}<\left(\nu\left(r_{m}, f\right)\right)^{k}(1+o(1))<r_{m}^{k} \exp \left\{-d r_{m}^{n}\right\}+o\left(r_{m}^{k}\right) .
$$

This is a contradiction. If $\delta=0$, then by (19), (20), (22), we obtain

$$
r_{m}^{k} \exp \left\{-d r_{m}^{n}\right\}+o\left(r_{m}^{k}\right)>\left(\nu\left(r_{m}, f\right)\right)^{k}>r_{m}^{M_{1}-1+k},
$$

a contradiction.

CASE (ii): $\theta_{0} \in S_{j}$ with $j$ even. Again there is $M_{3}>0$ such that $\theta_{m} \in S_{j}$ when $m>M_{3}$, and by Lemma 2.3 ,

$$
\operatorname{Re}\left\{P\left(r_{m} e^{i \theta_{m}}\right)\right\}>d r_{m}^{n},
$$

where $d=\alpha_{n}(1-n \varepsilon) \sin (n \varepsilon)>0$. Together with (20), for $\left\{z_{m}=r_{m} e^{i \theta_{m}}\right\}$, we deduce that

$$
\left(\nu\left(r_{m}, f\right)\right)^{k}>r_{m}^{k} \exp \left\{d r_{m}^{n}\right\}-o\left(r_{m}^{k}\right) .
$$

This implies that $\sigma_{2}(f) \geq n=\operatorname{deg}(P)$. Hence, $\sigma_{2}(f)=n=\operatorname{deg}(P)$.

CASE (iii): $\theta_{0}=-\vartheta_{n} / n+(2 j-1) \pi /(2 n)$ for some $j \in\{0,1, \ldots, 2 n-1\}$. Then, for the above $\varepsilon$, there exists some $N$ such that $\theta_{m} \in \bar{\Omega}_{1}=\left[\theta_{0}-\varepsilon, \theta_{0}+\varepsilon\right]$ and $z_{m} \in \bar{\Omega}=\left\{z: \theta_{0}-\varepsilon \leq \arg z \leq \theta_{0}+\varepsilon\right\}$ for $m>N$. If we fix a ray $\arg z=\theta \in \bar{\Omega}_{1} \backslash\left\{\theta_{0}\right\}$, then Lemma 2.3 shows that there is a positive constant $R$ such that $\operatorname{Re}\left\{P\left(r e^{i \theta}\right)\right\}>d_{1} r^{n}$ or $\operatorname{Re}\left\{P\left(r e^{i \theta}\right)\right\}<-d_{1} r^{n}$ for $r>R$, where $d_{1}$ is a suitable positive constant. A similar discussion to the one in the proof 
of Lemma 2.9, making use of Lemma 2.8 instead of Lemma 2.6, gives

$$
\left|f\left(r e^{i \theta}\right)\right| \leq(1+o(1)) 2\left|q_{s}\right| r^{k+\operatorname{deg}(Q)}, \quad \theta \in \bar{\Omega}_{1} \backslash\left\{\theta_{0}\right\},
$$

where $q_{s}$ is as above. However, noting that $z_{m} \in \bar{\Omega}(m>N),\left|f\left(z_{m}\right)\right|=$ $M\left(r_{m}, f\right)$ and that $f$ is of infinite order, we infer from (23) that $\theta_{m}=\theta_{0}$ for $m>N$, that is, $z_{m}=r_{m} e^{i \theta_{0}}$ for $m>N$. Together with $\theta_{0}=-\vartheta_{n} / n$ $+(2 j-1) \pi /(2 n)$, we have $\operatorname{Re}\left\{\alpha_{n} e^{i \vartheta_{n}}\left(r e^{i \theta_{0}}\right)^{n}\right\}=0$. From that and the expression of $P(z)$, we now have to consider the following two subcases.

Subcase (iii)(a): The $t$ mentioned above satisfies $\operatorname{Re}\left\{b_{n-1}\left(r e^{i \theta_{0}}\right)^{n-1}\right\}=$ $\cdots=\operatorname{Re}\left\{b_{t+1}\left(r e^{i \theta_{0}}\right)^{t+1}\right\}=0$ and $\operatorname{Re}\left\{b_{t}\left(r e^{i \theta_{0}}\right)^{t}\right\} \neq 0$, where $1 \leq t \leq n-1$. Then for sufficiently large $r_{m}$, we have $\operatorname{Re}\left\{P\left(r_{m} e^{i \theta_{m}}\right)\right\}=\operatorname{Re}\left\{P\left(r_{m} e^{i \theta_{0}}\right)\right\}>$ $d_{2} r_{m}^{t}$ or $\operatorname{Re}\left\{P\left(r_{m} e^{i \theta_{m}}\right)\right\}<-d_{2} r_{m}^{t}$, where $d_{2}$ is a suitable positive constant. If $\operatorname{Re}\left\{P\left(r_{m} e^{i \theta_{m}}\right)\right\}<-d_{2} r_{m}^{t}$, then by a similar argument to the proof of case (i), we find that for $\delta>0$,

$$
\exp \left\{k r_{m}^{\delta-\varepsilon}\right\}<\left(\nu\left(r_{m}, f\right)\right)^{k}(1+o(1))<r_{m}^{k} \exp \left\{-d_{2} r_{m}^{t}\right\}+o\left(r_{m}^{k}\right),
$$

and for $\delta=0$,

$$
r_{m}^{k(M-1)}<\left(\frac{\nu\left(r_{m}, f\right)}{r_{m}}\right)^{k}(1+o(1))<\exp \left\{-d_{2} r_{m}^{t}\right\}+o(1),
$$

two contradictions. If $\operatorname{Re}\left\{P\left(r_{m} e^{i \theta_{m}}\right)\right\}>d_{2} r_{m}^{t}$, then by a similar discussion to the proof of case (ii), we get

$$
\left(\nu\left(r_{m}, f\right)\right)^{k}(1+o(1))>r_{m} \exp \left\{d_{2} r_{m}^{t}\right\}-o(1) .
$$

This implies that $\sigma_{2}(f) \geq t \geq 1$. Hence $\sigma_{2}(f)=t$.

Subcase (iii)(b): $\operatorname{Re}\left\{b_{n-1}\left(r e^{i \theta_{0}}\right)^{n-1}\right\}=\cdots=\operatorname{Re}\left\{b_{1}\left(r e^{i \theta_{0}}\right)\right\}=0$. Then there exists some positive constant $M_{1}$ such that $-M_{1}<\operatorname{Re}\left\{P\left(r_{m} e^{i \theta_{0}}\right)\right\}$ $<M_{1}$, that is,

$$
e^{-M_{1}} \leq\left|e^{P\left(r_{m} e^{i \theta_{m}}\right)}\right|=\left|e^{P\left(r_{m} e^{i \theta_{0}}\right)}\right| \leq e^{M_{1}} .
$$

It follows from (18) (or (19)), (20) and (24) that

$$
\begin{aligned}
\frac{1}{r_{m}^{k}} \exp \left\{k r_{m}^{\delta-\varepsilon}\right\}-o(1) & \leq\left(\frac{\nu\left(r_{m}, f\right)}{r_{m}}\right)^{k}(1+o(1))-o(1) \\
& \leq\left|\exp \left\{P\left(r_{m} e^{i \theta_{m}}\right)\right\}\right| \leq e^{M_{1}},
\end{aligned}
$$

or

$r_{m}^{k(M-1)}-o(1) \leq\left(\frac{\nu\left(r_{m}, f\right)}{r_{m}}\right)^{k}(1+o(1))-o(1) \leq\left|\exp \left\{P\left(r_{m} e^{i \theta_{m}}\right)\right\}\right| \leq e^{M_{1}}$

two contradictions.

In conclusion, the hyperorder of $f$ is a positive integer not exceeding the degree of $P$ when $P$ is a polynomial. 
Secondly, suppose that $P$ is a transcendental entire function with $\sigma(P)$ $<1 / 2$. By Lemma 2.9 and (2), $f$ must be transcendental and of infinite order. Choose $z$ satisfying $|z|=r \notin[0,1] \cup E_{1}$ and $|f(z)|=M(r, f)$, where $E_{1}$ is a subset of $(1, \infty)$ of finite logarithmic measure. Then (17) also holds. Hence, we have

$$
e^{P(z)}=\left(\frac{\nu(r, f)}{z}\right)^{k}(1+o(1))+o(1) .
$$

Since $\sigma(P)<1 / 2$, Lemma 2.4 (or 2.5 ) yields a set $H \subset(1, \infty)$ of positive lower logarithmic density (hence, of infinite logarithmic measure) such that for all $|z|=r \in H$, we have $|P(z)| \geq M(r, P)^{c}$, where $c$ is a constant with $0<c<1$. Assume that the hyperorder of $f$ is finite. Then by Lemma 2.2, we have $\nu(r, f) \geq|z|^{M}$ for any positive constant $M$. Taking a principal branch of $\log \left((\nu(r, f) / z)^{k}(1+o(1))+o(1)\right)$, it follows from (25) that for all $z$ with $|z|=r \in H \backslash[0,1] \cup E_{1}$ and for any given $\varepsilon>0$,

$$
\begin{aligned}
M(r, P)^{c} & \leq|P(z)| \leq|\log |\left(\frac{\nu(r, f)}{z}\right)^{k}(1+o(1))+o(1)|| \\
& \leq k \log \nu(r, f)+O(1) \leq k r^{\sigma_{2}(f)+1}+O(1) .
\end{aligned}
$$

This contradicts the assumption that $P$ is transcendental. Therefore the hyperorder of $f$ must be infinite.

4. Proof of Theorem 1.3. Theorem 1.3 can be proved in much the same way as Theorem 1.2 by making use of Lemma 2.10 instead of Lemma 2.4 (or 2.5 ).

5. Application. We say that two nonconstant meromorphic functions $f$ and $g$ share a meromorphic function $h$ provided that $f(z)-h(z)=0$ if and only if $g(z)-h(z)=0$. The functions $f$ and $g$ share $h C M$ if $f-h$ and $g-h$ have the same zeros with the same multiplicities. Let us recall the conjecture of Brück [4]: Let $f$ be a nonconstant entire function whose hyperorder is finite but not a positive integer. If $f$ and $f^{\prime}$ share some finite value a $C M$, then $f^{\prime}-a=c(f-a)$, where $c$ is a nonzero constant. The conjecture has been partly confirmed under various conditions (see [4], [11], [7]). Here we extend these results to the case where $f$ shares a polynomial with its $k$ th derivative.

THEOREM 5.1. Let $f$ be a nonconstant entire function with hyperorder less than $1 / 2$. If $f$ and $f^{(k)}$ share a polynomial $R C M$, then $f^{(k)}-R=$ $c(f-R)$, where $c$ is a nonzero constant.

Proof. When $R$ is a constant, the theorem has been proved by Chen and Shon [6]. Now we assume that $R$ is a nonconstant polynomial. By the 
assumptions and the essential part of the factorization theorem for meromorphic functions of finite iterated order ([14, Satz 12.4]), we have

$$
\frac{f^{(k)}-R}{f-R}=e^{P(z)},
$$

where $P$ is an entire function with $\sigma(P)=\sigma_{2}\left(e^{P}\right)<1 / 2$.

If $P$ is a constant, then the conclusion immediate. Now we assume that $P$ is a nonconstant entire function with $\sigma(P)<1 / 2$. Set $F(z)=f(z)-R(z)$ and $Q(z)=R(z)+R^{(k)}(z)$. Then $(26)$ can be rewritten as

$$
F^{(k)}-e^{P(z)} F=Q(z) .
$$

By Theorem 1.2, the order of $F$, and hence of $f$, is infinite, and the hyperorder of $f$ is a positive integer or infinity. This contradicts the assumption that the hyperorder of $f$ is less than $1 / 2$.

Acknowledgements. The author would like to thank the referee for valuable suggestions and comments, and Professors Hong-Xun Yi and ZongXuan Chen for their instructive guidance and useful help.

This work was supported by the NNSF of China (No. 10771121), the NSF of Jiangxi of China (No. 2008GQS0075) and the SRFDP of China (No. 20060422049).

\section{References}

[1] S. Bank, A general theorem concerning the growth of solutions of first-order algebraic differential equations, Compos. Math. 25 (1972), 61-70.

[2] P. D. Barry, On a theorem of Besicovitch, Quart. J. Math. 14 (1963), 293-302.

[3] - Some theorems related to the $\cos \pi \rho$ theorem, Proc. London Math. Soc. 21 (1970), 334-360.

[4] R. Brück, On entire functions which share one value CM with their first derivative, Results Math. 30 (1996), 21-24.

[5] Z. X. Chen and S. A. Gao, The complex oscillation theory of certain non-homogeneous linear differential equations with transcendental entire coefficients, J. Math. Anal. Appl. 179 (1993), 403-416.

[6] Z. X. Chen and K. H. Shon, On the entire function sharing one value CM with $k$-th derivatives, J. Korean Math. Soc. 42 (2005), 85-99.

[7] - - - On conjecture of $R$. Brück concerning the entire function sharing one value CM with its derivative, Taiwanese J. Math. 8 (2004), 235-244.

[8] Z. X. Chen and C. C. Yang, Some further results on the zeros and growths of entire solutions of second order linear differential equations, Kodai Math. J. 22 (1999), $273-285$.

[9] Z. X. Chen and Z. L. Zhang, Entire functions sharing fixed points with their higher order derivatives, Acta Math. Sinica Chinese Ser. 50 (2007), 1213-1222.

[10] G. G. Gundersen, Estimates for the logarithmic derivative of a meromorphic function, plus similar estimates, J. London Math. Soc. 37 (1988), 88-104. 
[11] G. G. Gundersen and L. Z. Yang, Entire functions that share one value with one or two of their derivatives, J. Math. Anal. Appl. 223 (1998), 88-95.

[12] W. Hayman, Meromorphic Functions, Clarendon Press, Oxford 1964.

[13] Y. Z. He and X. Z. Xiao, Algebroid Functions and Ordinary Differential Equations, Science Press, Beijing, 1988 (in Chinese).

[14] G. Jank und L. Volkmann, Meromorphe Funktionen und Differentialgleichungen, Birkhäuser, 1985.

[15] I. Laine, Nevanlinna Theory and Complex Differential Equations, de Gruyter, Berlin, 1993.

[16] A. I. Markushevich, Theory of Functions of a Complex Variable, Vol. 2, PrenticeHall, Englewood Cliffs, NJ, 1965.

[17] L. R. Sons, An analogue of a theorem of W. H. J. Fuchs on gap series, Proc. London Math. Soc. 21 (1970), 525-539.

[18] J. P. Wang, Entire functions that share a polynomial with their derivatives, J. Math. Anal. Appl. 320 (2006), 703-717.

[19] L. Yang, Value Distribution Theory, Springer, Berlin, and Science Press, Beijing, 1993.

[20] L. Z. Yang, Solution of a differential equation and its applications, Kodai Math. J. 22 (1999), 458-464.

[21] —, Entire functions that share one value with one of their derivatives, in: Finite or Infinite Dimensional Complex Analysis (Fukuoka, 1999) Lecture Notes in Pure Appl. Math. 214, Dekker, New York, 2000, 617-624.

[22] - , The growth of linear differential equations and their applications, Israel J. Math. 147 (2005), 359-370.

[23] H. X. Yi and C.-C. Yang, Uniqueness Theory of Meromorphic Functions, Science Press, 1995, and Kluwer, 2003.

Department of Mathematics

Nanchang University

Nanchang, Jiangxi 330031, China

E-mail: tbcao@ncu.edu.cn, ctb97@163.com

Received 28.6.2008

and in final form 9.9.2008 